\title{
Positive Polynomials and Boundary Interpolation with Finite Blaschke Products
}

\author{
Sergei Kalmykov ${ }^{1,2}$ • Béla Nagy ${ }^{3,4}$
}

Received: 29 July 2021 / Revised: 24 September 2021 / Accepted: 4 October 2021 /

Published online: 14 December 2021

(c) The Author(s) 2021

\begin{abstract}
The famous Jones-Ruscheweyh theorem states that $n$ distinct points on the unit circle can be mapped to $n$ arbitrary points on the unit circle by a Blaschke product of degree at most $n-1$. In this paper, we provide a new proof using real algebraic techniques. First, the interpolation conditions are rewritten into complex equations. These complex equations are transformed into a system of polynomial equations with real coefficients. This step leads to a "geometric representation" of Blaschke products. Then another set of transformations is applied to reveal some structure of the equations. Finally, the following two fundamental tools are used: a Positivstellensatz by Prestel and Delzell describing positive polynomials on compact semialgebraic sets using Archimedean module of length $N$. The other tool is a representation of positive polynomials in a specific form due to Berr and Wörmann. This, combined with a careful calculation of leading terms of occurring polynomials finishes the proof.
\end{abstract}

Keywords Blaschke product · Boundary interpolation - Rational interpolation · Real algebraic geometry $\cdot$ Positivstellensatz

Mathematics Subject Classification 30E05 · 14P10 · 26C15

Communicated by Elias Wegert.

$\bowtie$ Béla Nagy

nbela@math.u-szeged.hu

Sergei Kalmykov

kalmykovsergei@sjtu.edu.cn

1 School of Mathematical Sciences, Shanghai Jiao Tong University, 800 Dongchuan Road, Shanghai 200240, People's Republic of China

2 Keldysh Institute of Applied Mathematics, Russian Academy of Sciences, Miusskaya pl., 4, 125047 Moscow, Russia

3 Alfréd Rényi Institute of Mathematics, Reáltanoda utca 13-15, 1053 Budapest, Hungary

4 ELKH-SZTE Analysis and Stochastics Research Group, Bolyai Institute, University of Szeged, Aradi vértanuk tere 1, 6720 Szeged, Hungary 


\section{Introduction}

Interpolating with Blaschke products has a large literature. In this paper, we focus exclusively on boundary interpolation, that is when we interpolate with Blaschke products and the nodes are on the unit circle. Necessarily, the values are on the unit circle too. The possibility of such interpolation was established in Cantor and Phelps [3]. Jones and Ruscheweyh [15] sharpened that result and showed that any $m$ pairs of data can be interpolated with a Blaschke product of degree at most $m-1$.

We denote the set of Blaschke products of degree $m$ by

$$
\mathbf{B}_{m}:=\left\{\gamma \prod_{j=1}^{m} \frac{z-a_{j}}{1-\overline{a_{j}} z}: \gamma, a_{1}, \ldots, a_{m} \in \mathbb{C},|\gamma|=1,\left|a_{1}\right|, \ldots,\left|a_{m}\right|<1\right\} .
$$

Here, $\mathbf{B}_{0}$ consists of constants (with modulus 1). We also write

$$
\mathbf{B}_{\leq m}:=\bigcup_{k=0}^{m} \mathbf{B}_{k}
$$

Theorem 1 (Jones-Ruscheweyh, 1987) Let $0 \leq \varphi_{1}<\varphi_{2}<\ldots<\varphi_{m}<2 \pi$ and $\psi_{1}, \psi_{2}, \ldots, \psi_{m} \in[0,2 \pi)$. Then there exists a Blaschke product $B \in \mathbf{B}_{\leq m-1}$ such that $B\left(\exp \left(i \phi_{j}\right)\right)=\exp \left(i \psi_{j}\right), j=1,2, \ldots, m$.

Actually, there are several proofs of this result and there is a very nice overview in the excellent paper [17] by Semmler and Wegert from 2006. Some earlier references are (not exhaustive list): [1,6-13,18,19] and it is also worth mentioning the books $[4,5]$.

The outline of the paper is the following. After this introduction, we present a new proof in detail. The proof consists of two main steps: first, we parametrize Blaschke products and transform the boundary Blaschke interpolation problem to interpolation of real values with special real rational functions. After this transformation, we have two sets: one coming from the Blaschke product representation, and another one coming from interpolation data. These sets are subsets of some high dimensional real euclidean space and the assertion of the Jones-Ruscheweyh theorem is equivalent to that their intersection is not empty. We show this by employing tools from real algebraic geometry. In particular, the main tool in this step is a Positivstellensatz of Prestel and Delzell. Finally, we present some technical lemmas and their proofs in Sect. 5. It would be interesting to compare our approach with that of Semmler and Wegert, and also investigate the structure of solutions as a future paper.

\section{First Part of the Proof}

In this part, we rephrase our problem completely. First, we apply the Cayley transform on finite Blaschke products. Let us mention that Semmler and Wegert used this approach, and they established a natural description (see [17, Lem. 3]). As we need 
the exact dependence of the coefficients on the zeros of the Blaschke product, we detail this step. In this way, we transform Blaschke products (of degree at most $m$, denoted by $\mathbf{B}_{\leq m}$ ) to a special subset of real rational functions (denoted by $\mathbf{H}_{m}$ ). Here the coordinates represent the coefficients, and we use a natural choice to exclude the ambiguity (caused by multiplying the numerator and the denominator by the same constant). We also transform the interpolation data and this yields a homogeneous set of data (S, solution set). If the number of interpolation pairs $(n)$ and the degree of Blaschke products $(\leq m)$ differ by one only (i.e. $n=m+1)$, then there is a nice description of the solution set $\mathbf{S}$. Formulating this description (linear parametrization of $\mathbf{S}$ ) finishes the rephrasing of the problem to real algebraic equations and it is the last step of the first part.

\subsection{Parametrizing the Blaschke Products}

We use the unit disk $\mathbb{D}:=\{z \in \mathbb{C}:|z|<1\}$ and the unit circle $\mathbb{T}:=\{z \in \mathbb{C}:|z|=1\}$. We introduce a parametrization as follows. Let

$$
\begin{aligned}
& \mathbf{E}_{m}:=\mathbb{T} \times \mathbb{D}^{m} \subset \mathbb{C}^{m+1}, \\
& \mathbf{F}_{m}:=\mathbb{T} \times(\mathbb{D} \cup \mathbb{T})^{m} \subset \mathbb{C}^{m+1}
\end{aligned}
$$

where the closure of $\mathbf{E}_{m}$ is $\mathbf{F}_{m}$,

Now we investigate how $\mathbf{E}_{m}$ and $\mathbf{F}_{m}$ can be used to parametrize Blaschke products. Consider the parametrization mapping

$$
\mathcal{P}_{m}: \mathbf{F}_{m} \rightarrow \mathbf{B}_{\leq m}, \quad\left(\delta, a_{1}, \ldots, a_{m}\right) \mapsto \delta^{2} \prod_{j:\left|a_{j}\right|<1} \frac{z-a_{j}}{1-\overline{a_{j}} z} \prod_{j:\left|a_{j}\right|=1}\left(-a_{j}\right)
$$

where we set

$$
\gamma=\delta^{2}
$$

for convenience (see (10), (11) and Lemma 1 below). Note that if $a \rightarrow a_{0},|a|<1$, $\left|a_{0}\right|=1$, then $(z-a) /(1-\bar{a} z) \rightarrow-a_{0}$ locally uniformly in $\mathbb{D}$, and also on $\mathbb{D} \cup \mathbb{T}$. Hence, $\mathcal{P}_{m}$ is a continuous mapping (from the euclidean topology to the locally uniform convergence topology), see also Lemma 3. Roughly speaking, $\mathcal{P}_{m}$ maps $\mathbf{E}_{m}$, and $\mathbf{F}_{m}$ to Blaschke products of degree $m$ and Blaschke products of degree at most $m$. More precisely,

$$
\mathcal{P}_{m}\left(\mathbf{E}_{m}\right)=\mathbf{B}_{m} \text { and } \mathcal{P}_{m}\left(\mathbf{F}_{m}\right)=\mathbf{B}_{\leq m}
$$

\subsection{The Cayley Transform}

In this section, we give a description of how the Cayley transform behaves on Blaschke products of degree at most $m$. We also need the inverse Cayley transform too. We denote 
the Cayley transform by $z=T(u)$ :

$$
\begin{gathered}
z=T(u)=\frac{i-u}{i+u}, \\
u=T^{-1}(z)=i \frac{1-z}{1+z} .
\end{gathered}
$$

The set of real rational functions of degree at most $m$ is:

$$
\mathbf{H}_{m}:=\left\{\frac{P(u)}{Q(u)}: P(u), Q(u) \in \mathbb{R}[u], \operatorname{deg}(P), \operatorname{deg}(Q) \leq m, Q \not \equiv 0\right\}
$$

It is important that if $B \in \mathbf{B}_{\leq m}$ is a Blaschke product, then

$$
H(u):=T^{-1} \circ B \circ T(u)=\frac{P(u)}{Q(u)}
$$

for some polynomials $P, Q$ with real coefficients and $\operatorname{deg}(P) \operatorname{deg}(Q) \leq m$. This follows immediately from [17, Lem. 3] (Representation Lemma) and we reprove and investigate it in detail in the next subsection.

\subsection{Structure of the Coefficients After the Cayley Transform}

We also need the structure of the coefficients of $P$ and $Q$ when $B$ is a Blaschke product $(|\gamma|=1)$ later, so we detail this calculation.

So let $B \in \mathbf{B}_{m}$ and we write

$$
\begin{aligned}
H(u) & =T^{-1}(B(T(u)))=i \frac{1-\gamma \prod_{j=1}^{m} \frac{z-a_{j}}{1-\overline{a_{j}} z}}{1+\gamma \prod_{j=1}^{m} \frac{z-a_{j}}{1-\overline{a_{j}} z}} \\
& =i \frac{\prod_{j}\left(1-\overline{a_{j}} z\right)-\gamma \prod_{j}\left(z-a_{j}\right)}{\prod_{j}\left(1-\overline{a_{j}} z\right)+\gamma \prod_{j}\left(z-a_{j}\right)} \\
& =i \frac{\prod_{j} \frac{u\left(1+\overline{a_{j}}\right)+i-\overline{a_{j}} i}{i+u}-\gamma \prod_{j} \frac{u\left(-1-a_{j}\right)+i-a_{j} i}{i+u}}{\prod_{j} \frac{u\left(1+\overline{a_{j}}\right)+i-\overline{a_{j}} i}{i+u}+\gamma \prod_{j} \frac{u\left(-1-a_{j}\right)+i-a_{j} i}{i+u}} \\
& =i \frac{\prod_{j}\left(u\left(1+\overline{a_{j}}\right)+i-\overline{a_{j}} i\right)-\gamma \prod_{j}\left(u\left(-1-a_{j}\right)+i-a_{j} i\right)}{\prod_{j}\left(u\left(1+\overline{a_{j}}\right)+i-\overline{a_{j}} i\right)+\gamma \prod_{j}\left(u\left(-1-a_{j}\right)+i-a_{j} i\right)} .
\end{aligned}
$$


To simplify this, we introduce

$$
\begin{aligned}
& C(u):=\prod_{j=1}^{m}\left(u\left(1+\overline{a_{j}}\right)+i-\overline{a_{j}} i\right) \\
& D(u):=\prod_{j=1}^{m}\left(u\left(-1-a_{j}\right)+i-a_{j} i\right)
\end{aligned}
$$

so we can write

$$
H(u)=\frac{i C(u)-i \gamma D(u)}{C(u)+\gamma D(u)} .
$$

Denote the coefficients of $C$ and $D$ by $c_{j}$ and $d_{j}$ respectively:

$$
C(u)=\sum_{j=0}^{m} c_{j} u^{j}, \quad D(u)=\sum_{j=0}^{m} d_{j} u^{j}
$$

Note that

$$
d_{j}=d_{j}\left(a_{1}, \ldots, a_{m}\right) \in \mathbb{C}\left[a_{1}, \ldots, a_{m}\right]
$$

are (holomorphic) polynomials in $a_{1}, \ldots, a_{m}$.

We also have $\overline{D(u)}=(-1)^{m} C(u)$ (when $u \in \mathbb{R}$ ), therefore

$$
c_{j}=(-1)^{m} \overline{d_{j}}
$$

for $j=0,1, \ldots, m$. We can express the leading coefficients, $c_{m}=\prod_{j=1}^{m}\left(1+\overline{a_{j}}\right)$ and $d_{m}=\prod_{j=1}^{m}\left(-1-a_{j}\right)$.

We are going to express $P$ and $Q$ using $C, D$ and (7). First, the leading coefficient of $i C(u)-i \gamma D(u)$ is

$$
i c_{m}-i \gamma d_{m}=i(-1)^{m} \overline{d_{m}}-i \gamma d_{m}
$$

and the leading coefficient of $C(u)+\gamma D(u)$ is

$$
c_{m}+\gamma d_{m}=(-1)^{m} \overline{d_{m}}+\gamma d_{m} .
$$


We use Lemma 1 (with $W=(-1)^{m} d_{m}$ ) since $|\gamma|=1$ and Lemma 2; Lemmas 1 and 2 can be found in Sect. 5. This way we get that

$$
\begin{aligned}
& P(u)=\left(\frac{1}{\sqrt{\gamma}} C-\sqrt{\gamma} D\right) \cdot \begin{cases}1, & \text { if } m \text { is odd, } \\
i, & \text { if } m \text { is even, }\end{cases} \\
& Q(u)=\left(\frac{1}{\sqrt{\gamma}} C+\sqrt{\gamma} D\right) \cdot \begin{cases}-i, & \text { if } m \text { is odd, } \\
1, & \text { if } m \text { is even }\end{cases}
\end{aligned}
$$

are polynomials with real coefficients and

$$
H(u)=\frac{P(u)}{Q(u)}
$$

Let us remark here that $P(u)$ and $Q(u)$ cannot have common zeros. Otherwise, if $u \in \mathbb{R}$ is such that $P(u)=0$ and $Q(u)=0$, then $C(u)=0$ and $D(u)=0$ too. Considering the definitions of $C(u)$ and $D(u)$, there should be $a_{j}, a_{k}$ with $\left|a_{j}\right|<1$, $\left|a_{k}\right|<1$ and $i\left(\overline{a_{j}}-1\right) /\left(\overline{a_{j}}+1\right)=i\left(a_{k}-1\right) /\left(-a_{k}-1\right)$, a contradiction. This is also in accord with [17, Rem. 1].

Up to now, any branch of the square root can be used $( \pm \sqrt{\gamma})$. Now using (4), hence $\sqrt{\gamma}=\delta$, we use $\delta$ instead below.

Continuing the calculation for the coefficients of $P(u)=\sum_{j=0}^{m} p_{j} u^{j}$ (and using $|\delta|=1$ again), we have

$$
p_{j}=\left(\frac{1}{\delta} c_{j}-\delta d_{j}\right) \cdot\left\{\begin{array}{ll}
1 \\
i
\end{array}= \begin{cases}-2 \operatorname{Re}\left(\delta d_{j}\right), & \text { if } m \text { is odd } \\
2 \operatorname{Im}\left(\delta d_{j}\right), & \text { if } m \text { is even }\end{cases}\right.
$$

The coefficients of $Q(u)=\sum_{j=0}^{m} q_{j} u^{j}$ are

$$
q_{j}=\left(\frac{1}{\delta} c_{j}+\delta d_{j}\right) \cdot\left\{\begin{array}{ll}
-i \\
1
\end{array}= \begin{cases}2 \operatorname{Im}\left(\delta d_{j}\right), & \text { if } m \text { is odd } \\
2 \operatorname{Re}\left(\delta d_{j}\right), & \text { if } m \text { is even }\end{cases}\right.
$$

\subsection{Parametrization of the Real Rational Functions}

We investigate the parametrization of rational functions. In the previous section, $p_{j}$ 's and $q_{j}$ 's are polynomials which are the coefficients of rational functions depending on the zeros of the Blaschke product. In this paragraph, we use $p_{j}$ 's and $q_{j}$ 's as variables which are the coefficients of rational functions for convenience (and slightly abusing the notation). Afterward, we use them again as polynomials. Let

$$
\begin{aligned}
& \mathbf{A}:=\mathbb{R}^{2 m+2}, \\
& \mathbf{A}_{1}:=\left\{\left(p_{m}, \ldots, p_{1}, p_{0}, q_{m}, \ldots, q_{1}, q_{0}\right) \in \mathbf{A}: q_{m}^{2}+\cdots+q_{1}^{2}+q_{0}^{2} \neq 0\right\} .
\end{aligned}
$$


It is standard that

$$
\left(p_{m}, \ldots, p_{1}, p_{0}, q_{m}, \ldots, q_{1}, q_{0}\right) \in \mathbf{A}_{1} \mapsto H(u)=\frac{\sum_{j=0}^{m} p_{j} u^{j}}{\sum_{j=0}^{m} q_{j} u^{j}} \in \mathbf{H}_{m}
$$

is a surjective but not bijective mapping, and if $\mathbf{p} \in \mathbf{A}_{1} \subset \mathbb{R}^{2 m+2}$, then $c \mathbf{p}$ determines the same rational function ( $c \in \mathbb{R} \backslash\{0\}$ ). In other words, the coefficients of the numerator and denominator of a rational function from $\mathbf{H}_{m}$ are not uniquely determined (unless some type of normalization is imposed on the denominator and numerator).

Therefore we directly define the coefficients

$$
\begin{array}{r}
\mathcal{L}_{m, \mathbb{C}}: \mathbf{F}_{m} \rightarrow \mathbf{A}, \quad\left(\delta, a_{1}, \ldots, a_{m}\right) \mapsto\left(p_{m}, \ldots, p_{1}, p_{0}, q_{m}, \ldots, q_{1}, q_{0}\right), \\
\text { where if } m \text { is odd, then } p_{j}=-2 \operatorname{Re}\left(\delta d_{j}\right), q_{j}=2 \operatorname{Im}\left(\delta d_{j}\right), \\
\text { and if } m \text { is even, then } p_{j}=2 \operatorname{Im}\left(\delta d_{j}\right), q_{j}=2 \operatorname{Re}\left(\delta d_{j}\right),
\end{array}
$$

where we used (12), (13) and (6). We know that

$$
d_{j}=\sum_{J \subset\{1, \ldots, m\},|J|=j}\left(\prod_{k \in J}-1-a_{k}\right)\left(\prod_{\ell \in J^{c}} i-a_{\ell} i\right)
$$

where $J^{c}=\{1,2, \ldots, m\} \backslash J$; in particular, if $j=0$, then $J=\emptyset$ and the sum consists of only one term, hence

$$
d_{0}=\prod_{\ell=1}^{m} i-a_{\ell} i
$$

Obviously, $\mathcal{L}_{m, \mathbb{C}}\left(\mathbf{F}_{m}\right) \subset \mathbf{A}_{1}$.

Finally, we switch to real algebraic language, in particular, we use new variables as follows

$$
\begin{aligned}
a_{j} & =x_{j}+i y_{j}, \text { where } x_{j}^{2}+y_{j}^{2} \leq 1, x_{j}, y_{j} \in \mathbb{R}, j=1, \ldots, m, \\
\delta & =\delta_{1}+i \delta_{2}, \text { where } \delta_{1}^{2}+\delta_{2}^{2}=1, \delta_{1}, \delta_{2} \in \mathbb{R} .
\end{aligned}
$$

With these substitutions, we introduce

$$
\begin{aligned}
\mathbf{F}_{m, \mathbb{R}}:=\left\{\left(\delta_{1}, \delta_{2}, x_{1}, y_{1}, \ldots, x_{m}, y_{m}\right) \in \mathbb{R}^{2 m+2}:\right. \\
\left.\delta_{1}^{2}+\delta_{2}^{2}=1, x_{j}^{2}+y_{j}^{2} \leq 1, j=1, \ldots, m\right\}
\end{aligned}
$$

and

$$
\mathcal{L}_{m}: \mathbf{F}_{m, \mathbb{R}} \rightarrow \mathbf{A}
$$

and as above, $\mathcal{L}_{m}\left(\mathbf{F}_{m, \mathbb{R}}\right) \subset \mathbf{A}_{1}$. 
Let $U_{\ell}$ and $V_{\ell}$ be the following polynomials

$$
\mathcal{L}_{m}:\left(\delta_{1}, \delta_{2}, x_{1}, y_{1}, \ldots, x_{m}, y_{m}\right) \mapsto\left(U_{m}, \ldots, U_{1}, U_{0}, V_{m}, \ldots, V_{1}, V_{0}\right)
$$

where $U_{\ell}, V_{\ell} \in \mathbb{R}\left[\delta_{1}, \delta_{2}, x_{1}, y_{1}, \ldots, x_{m}, y_{m}\right]$, and actually

$$
\begin{aligned}
& U_{\ell}\left(\delta_{1}, \delta_{2}, x_{1}, y_{1}, \ldots, x_{m}, y_{m}\right)=p_{\ell}\left(\delta_{1}+i \delta_{2}, x_{1}+i y_{1}, \ldots, x_{m}+i y_{m}\right), \\
& V_{\ell}\left(\delta_{1}, \delta_{2}, x_{1}, y_{1}, \ldots, x_{m}, y_{m}\right)=q_{\ell}\left(\delta_{1}+i \delta_{2}, x_{1}+i y_{1}, \ldots, x_{m}+i y_{m}\right),
\end{aligned}
$$

for $\ell=0,1, \ldots, m$. Note that for $\ell=0,1, \ldots, m$

$$
U_{\ell}= \begin{cases}-2 \delta_{1} \operatorname{Re}\left(d_{\ell}\right)+2 \delta_{2} \operatorname{Im}\left(d_{\ell}\right), & \text { if } m \text { is odd } \\ 2 \delta_{1} \operatorname{Im}\left(d_{\ell}\right)+2 \delta_{2} \operatorname{Re}\left(d_{\ell}\right), & \text { if } m \text { is even }\end{cases}
$$

and

$$
V_{\ell}= \begin{cases}2 \delta_{1} \operatorname{Im}\left(d_{\ell}\right)+2 \delta_{2} \operatorname{Re}\left(d_{\ell}\right), & \text { if } m \text { is odd } \\ 2 \delta_{1} \operatorname{Re}\left(d_{\ell}\right)-2 \delta_{2} \operatorname{Im}\left(d_{\ell}\right), & \text { if } m \text { is even }\end{cases}
$$

where $d_{\ell}$ depends on $a_{1}, \ldots, a_{m}$ while $U_{\ell}$ and $V_{\ell}$ depend on $x_{1}, y_{1}, \ldots, x_{m}, y_{m}$ but they are connected by (15).

\subsection{Applying Cayley Transform on the Interpolation Data}

Here we consider the interpolation data and transform it with the Cayley transform.

Suppose that pairwise distinct $z_{1}, \ldots, z_{n} \in \mathbb{C}$ with $\left|z_{1}\right|=\ldots=\left|z_{n}\right|=1$ are given and $w_{1}, \ldots, w_{n} \in \mathbb{C}$ with $\left|w_{1}\right|=\ldots=\left|w_{n}\right|=1$ are also given. We transform these and consider

$$
u_{j}:=T^{-1}\left(z_{j}\right), \quad v_{j}:=T^{-1}\left(w_{j}\right) .
$$

Note that if $w_{j}=-1$ for some $j$, then $v_{j}=\infty$; also if $z_{j}=-1$ for some $j$, then $u_{j}=$ $\infty$. By appropriate rotations, this can be avoided. To be precise, let $\omega, \chi \in \mathbb{C},|\omega|=$ $|\chi|=1$ such that none of $\omega w_{1}, \ldots, \omega w_{n}$ is equal to -1 and none of $z_{1} \chi, \ldots, z_{n} \chi$ is equal to -1 . Then we find a Blaschke product $B(\cdot)$ so that $B\left(z_{j} \chi\right)=\omega w_{j}$ for $j=1, \ldots, n$ where

$$
B(z)=\gamma \prod_{j=1}^{m} \frac{z-a_{j}}{1-\overline{a_{j}} z}
$$

Then just take

$$
\widetilde{B}(z):=\omega^{-1} \gamma \chi^{-m} \prod_{j=1}^{m} \frac{z-a_{j} \chi}{1-\overline{a_{j} \chi} z}
$$


and this will interpolate $w_{j}$ at $z_{j}: \widetilde{B}\left(z_{j}\right)=w_{j}, j=1, \ldots, m$.

Therefore, we may assume that all $u_{j}$ and $v_{j}$ are finite, i.e.

$$
\begin{array}{r}
u_{1}, \ldots, u_{n}, v_{1}, \ldots, v_{n} \in \mathbb{R}, \\
u_{1}, \ldots, u_{n} \text { are pairwise distinct. }
\end{array}
$$

\subsection{Introducing Two Real Sets Coming from Blaschke Products and Interpolation Data}

Here we would like to find real polynomials

$$
P(u)=\sum_{\ell=0}^{m} \alpha_{\ell} u^{\ell} \text { and } Q(u)=\sum_{\ell=0}^{m} \beta_{\ell} u^{\ell},
$$

$Q(u) \not \equiv 0$ such that

$$
P\left(u_{j}\right)-v_{j} Q\left(u_{j}\right)=0, j=1, \ldots, n
$$

This equation is equivalent to $P\left(u_{j}\right) / Q\left(u_{j}\right)=v_{j}$, provided that

$$
Q\left(u_{j}\right) \neq 0 \text {. }
$$

We will return to this condition later.

An equivalent form of (22) is

$$
\sum_{\ell=0}^{m}\left(\alpha_{\ell}-v_{j} \beta_{\ell}\right) u_{j}^{\ell}=0, j=1, \ldots, n .
$$

Note that this is a homogeneous linear equation in $\alpha_{0}, \beta_{0}, \ldots, \alpha_{m}, \beta_{m}$, so it always has a solution. Let $\mathbf{U}$ be the following Vandermonde matrix

$$
\mathbf{U}:=\left(u_{j}^{\ell}\right)_{j=1,2, \ldots, n ; \ell=0,1, \ldots, m}=\left(\begin{array}{cccc}
1 & u_{1} & \ldots & u_{1}^{m} \\
1 & u_{2} & \ldots & u_{2}^{m} \\
\vdots & & & \\
1 & u_{n} & \ldots & u_{n}^{m}
\end{array}\right),
$$

and put $\mathbf{D}:=\operatorname{diag}\left(-v_{1}, \ldots,-v_{n}\right)$ and $\mathbf{v}:=\left(\alpha_{0}, \alpha_{1}, \ldots, \alpha_{m}, \beta_{0}, \beta_{1}, \ldots, \beta_{m}\right)^{\top}$ (where.$^{\top}$ means transpose hence $\mathbf{v}$ is a column vector) and introduce

$$
\mathbf{M}:=(\mathbf{U} \mathbf{D U}) \in \mathbb{R}^{n \times(2 m+2)}
$$

for short. Hence, (24) can be written as

$$
\mathbf{M v}=0 .
$$


We assume that

$$
m=n-1 \text {. }
$$

So $\mathbf{U}$ is a square matrix, $\mathbf{U} \in \mathbb{R}^{n \times n}$, and since $u_{1}, \ldots, u_{n}$ are pairwise different (see (21)), $\mathbf{U}$ is a non-singular matrix. Note that $\mathbf{M}$ can be thought of as a mapping from $\mathbf{A}=\mathbb{R}^{2 m+2}$. Therefore,

$$
\operatorname{rank}(\mathbf{M})=n \text {. }
$$

Using the rank-nullity theorem, this implies that

$$
\mathbf{S}:=\operatorname{ker}(\mathbf{M}) \text { has dimension } n \text {. }
$$

Note that $\mathbf{S}=\mathbf{S}\left(u_{1}, \ldots, u_{n}, v_{1}, \ldots, v_{n}\right) \subset \mathbf{A}$ depends on the interpolation data (i.e. on $z_{1}, \ldots, z_{n}$ and $w_{1}, \ldots, w_{n}$ ) and contains all the solutions of (24) while the "geometrical representation" of general Blaschke products,

$$
\mathbf{G}_{\leq m}:=\mathcal{L}_{m}\left(\mathbf{F}_{m, \mathbb{R}}\right)
$$

is independent of the interpolation data.

We return to the condition (23). It holds because

$$
\mathbf{v}=\left(\alpha_{0}, \alpha_{1}, \ldots, \alpha_{m}, \beta_{0}, \beta_{1}, \ldots, \beta_{m}\right) \in \mathbf{G}_{\leq m}
$$

hence the corresponding $P(u)=\sum_{j=0}^{m} \alpha_{j} u^{j}, Q(u)=\sum_{j=0}^{m} \beta_{j} u^{j}$ satisfy that $P(u) / Q(u)=T^{-1} \circ B \circ T(u)$ holds for some Blaschke product $B \in \mathbf{B}_{\leq m}$. Therefore $P$ and $Q$ cannot have common zeros.

Note that $\mathbf{G}_{\leq m} \subset \mathbf{A}_{1}$, moreover

$$
\begin{aligned}
\mathbf{G}_{\leq m} \subset\{ & \left(p_{m}, \ldots, p_{1}, p_{0}, q_{m}, \ldots, q_{1}, q_{0}\right) \in \mathbf{A}: \\
& \left.p_{m}^{2}+\ldots+p_{1}^{2}+p_{0}^{2} \neq 0, q_{m}^{2}+\ldots+q_{1}^{2}+q_{0}^{2} \neq 0\right\} .
\end{aligned}
$$

Also, observe that $\mathbf{S} \backslash\{0\} \subset \mathbf{A}_{1}$. Indeed, if $\mathbf{v} \in \mathbf{S} \backslash\{0\}$ and $\mathbf{v} \in \mathbf{A} \backslash \mathbf{A}_{1}=\mathbf{A}_{1}^{c}$, then $\mathbf{v}$ has the form $\left(\alpha_{1}, \ldots, \alpha_{n}, 0, \ldots, 0\right)$ where $\left(\alpha_{1}, \ldots, \alpha_{n}\right) \neq 0$. Substituting this into (24), we get that $\mathbf{U}\left(\alpha_{1}, \ldots, \alpha_{n}\right)^{\top}=0$ which contradicts the fact that $\mathbf{U}$ is invertible.

It is straightforward to see that the Jones-Ruscheweyh theorem is equivalent to

$$
\mathbf{S} \cap \mathbf{G}_{\leq m} \neq \emptyset .
$$

\subsection{Parametrizing the Set Coming from Interpolation Data}

We now parametrize $\mathbf{S}$. In the previous section, $\alpha_{j}$ 's and $\beta_{j}$ 's were unknowns, coming from a subspace: $\left(\alpha_{0}, \alpha_{1}, \ldots, \alpha_{m}, \beta_{0}, \beta_{1}, \ldots, \beta_{m}\right) \in \mathbf{S}$. As we parametrize $\mathbf{S}$, we 
use the same symbols: $\alpha_{j}=\alpha_{j}\left(t_{1}, \ldots, t_{n}\right), \beta_{j}=\beta_{j}\left(t_{1}, \ldots, t_{n}\right)$ to keep the notation simple. Using $t=\left(t_{1}, \ldots, t_{n}\right) \in \mathbb{R}^{n}$, we consider

$$
t \in \mathbb{R}^{n} \mapsto\left(\alpha_{0}(t), \ldots, \alpha_{m}(t), \beta_{0}(t), \ldots, \beta_{m}(t)\right) \in \mathbf{A}=\mathbb{R}^{2 m+2}
$$

where $\alpha_{0}, \alpha_{1}, \ldots, \alpha_{m}$ and $\beta_{0}, \beta_{1}, \ldots, \beta_{m}$ are linear polynomials without constant terms:

$$
\alpha_{0}(0, \ldots, 0)=\ldots=\alpha_{m}(0, \ldots, 0)=\beta_{0}(0, \ldots, 0)=\ldots=\beta_{m}(0, \ldots, 0)=0
$$

Note that (29) is equivalent to that the system

$$
\left.\begin{array}{l}
U_{\ell}\left(\delta_{1}, \delta_{2}, x_{1}, y_{1}, \ldots, x_{m}, y_{m}\right)=\alpha_{\ell}\left(t_{1}, \ldots, t_{n}\right), \\
V_{\ell}\left(\delta_{1}, \delta_{2}, x_{1}, y_{1}, \ldots, x_{m}, y_{m}\right)=\beta_{\ell}\left(t_{1}, \ldots, t_{n}\right), \quad \ell=0,1, \ldots, m
\end{array}\right\}
$$

has a solution under the conditions

$$
x_{j}^{2}+y_{j}^{2} \leq 1, j=1, \ldots, m, \quad \delta_{1}^{2}+\delta_{2}^{2}=1
$$

where $\delta_{1}, \delta_{2}, x_{1}, y_{1}, \ldots, x_{m}, y_{m}, t_{1}, \ldots, t_{n} \in \mathbb{R}$.

This reformulation is expressed in terms of real algebraic geometry only.

To exploit the dimension condition (28), we introduce the matrix $A$ by collecting the coefficients of $\alpha_{0}, \alpha_{1}, \ldots, \alpha_{m}, \beta_{0}, \beta_{1}, \ldots, \beta_{m}$. Let $A \in \mathbb{R}^{2 n \times n}$ be the matrix for which

$$
A t^{\top}=\left(\begin{array}{c}
\alpha_{0}(t) \\
\beta_{0}(t) \\
\alpha_{1}(t) \\
\beta_{1}(t) \\
\vdots \\
\alpha_{m}(t) \\
\beta_{m}(t)
\end{array}\right)
$$

where $t^{\top}$ is a column vector. Now we use the dimension condition (28). Hence, it is standard (see e.g. [14, p. 13,0.4.6 (f)]) that there is an invertible matrix $B_{1} \in \mathbb{R}^{n \times n}$ and a set $I_{1} \subset\{1,2, \ldots, 2 n\}$ where $\left|I_{1}\right|=n$ such that the rows of $A B_{1}$ with indices from $I_{1}$ is the identity matrix of size $n$.

For ease of notation, we do not introduce new variables for $B_{1}^{-1} t^{\top}$, that is, we assume that the rows of $A$ with indices from $I_{1}$ give the identity matrix. For simplicity, we label the set of $I_{1}$ with $j():. j(1), \ldots, j(n)$ are distinct, $I_{1}=\{j(1), \ldots, j(n)\}$. Put $I_{2}:=\{1,2, \ldots, 2 n\} \backslash I_{1}$ for the remaining row indices. 
For convenience, we introduce

$$
\left.\begin{array}{l}
L_{2 \ell+1}(t):=\alpha_{\ell}(t) \\
L_{2 \ell+2}(t):=\beta_{\ell}(t)
\end{array}\right\}
$$

for $\ell=0,1, \ldots, m$. Therefore

$$
L_{j(k)}=t_{k}, \quad k=1,2, \ldots, n
$$

\section{A Second Transformation Applied on the Two Sets}

Our ultimate goal is to show that the two sets ( $\mathbf{S}$ and $\mathbf{G}_{\leq m}$ ) coming from different "sides" of the problem have non-empty intersection, i.e (29) holds.

In this section, we apply a second transformation which is an adapted form of the rational parametrization of the unit circle. This transformation changes the occurring polynomials and reveals a crucial property of the polynomials (see (44), (45), and (46)). We exploit this property with a Positivstellensatz of Delzell and Prestel which is a special description of positive polynomials on compact, semialgebraic sets. Instead of sums of squares of polynomials, it features higher powers of polynomials.

\subsection{Describing the Second Transformation}

We will transform our system of equations by substituting variables and replacing equations so that we can apply a Positivstellensatz.

It is known that

$$
\tau \mapsto\left(\frac{1-\tau^{2}}{1+\tau^{2}}, \frac{2 \tau}{1+\tau^{2}}\right)
$$

is a rational parametrization of the unit circle, more precisely, it is a bijective mapping from $\mathbb{R}$ to $\left\{(x, y) \in \mathbb{R}^{2}: x^{2}+y^{2}=1\right\} \backslash\{(-1,0)\}$. Similarly,

$$
(\sigma, r) \mapsto\left(\frac{1-\sigma^{2}}{1+\sigma^{2}}, \frac{2 r \sigma}{1+\sigma^{2}}\right)
$$

is a rational mapping from $(\sigma, r) \in[0, \infty) \times[-1,1]$ to $\left\{(x, y) \in \mathbb{R}^{2}: x^{2}+y^{2} \leq\right.$ $1\} \backslash\{(-1,0)\}$ and bijective from $(0, \infty) \times[-1,1]$ to $\left\{(x, y) \in \mathbb{R}^{2}: x^{2}+y^{2} \leq\right.$ $1\} \backslash\{(-1,0),(1,0)\}$ and $\{0\} \times[-1,1]$ is mapped to the point $(1,0)$. We also use $\sigma=s /(1-s)$ and $\tau=u /\left(1-u^{2}\right)$, so if $s$ runs over the interval $(0,1)$, then $\sigma$ runs over the interval $(0, \infty)$ and if $u$ runs over the interval $(-1,1)$, then $\tau$ runs over the real numbers. Therefore we consider the composite mapping:

$$
u \mapsto\left(\frac{u^{4}-3 u^{2}+1}{u^{4}-u^{2}+1},-\frac{2 u\left(u^{2}-1\right)}{u^{4}-u^{2}+1}\right)
$$


which is a bijective mapping from the open interval $(-1,1)$ to $\left\{(x, y) \in \mathbb{R}^{2}: x^{2}+y^{2}=\right.$ $1\} \backslash\{(-1,0)\}$ and maps -1 and +1 to the point $(-1,0)$. Similarly, we consider

$$
(s, r) \mapsto\left(\frac{1-2 s}{1-2 s+2 s^{2}},-\frac{2 r s(-1+s)}{1-2 s+2 s^{2}}\right)
$$

which has the following mapping properties. It maps $[0,1] \times(-1,1)$ bijectively to the open unit disk $\left\{(x, y) \in \mathbb{R}^{2}: x^{2}+y^{2}<1\right\}$, it is also bijective mapping from $(0,1) \times[-1,1]$ to $\left\{(x, y) \in \mathbb{R}^{2}: x^{2}+y^{2} \leq 1\right\} \backslash\{(1,0),(-1,0)\}$, maps $\{1\} \times[-1,1]$ to the point $(-1,0)$ and maps $\{0\} \times[-1,1]$ to the point $(1,0)$.

Based on these, we introduce the following substitutions:

$$
\begin{array}{r}
x_{j}=\frac{1-2 s_{j}}{1-2 s_{j}+2 s_{j}^{2}}, \quad y_{j}=-\frac{2 r_{j} s_{j}\left(-1+s_{j}\right)}{1-2 s_{j}+2 s_{j}^{2}}, \quad j=1,2, \ldots, m, \\
\delta_{1}=\frac{u^{4}-3 u^{2}+1}{u^{4}-u^{2}+1}, \quad \delta_{2}=-\frac{2 u\left(u^{2}-1\right)}{u^{4}-u^{2}+1} .
\end{array}
$$

We will apply these substitutions and to obtain polynomials, we will multiply them by the denominators. To see it precisely, we focus on the structure of $d_{\ell}$ and use formula (14) with the substitutions (15), (16) and the substitutions above so we can write for $\ell=0,1, \ldots, m$

$$
\begin{aligned}
d_{\ell}= & \sum_{J:|J|=\ell}(-1)^{\ell} i^{m-\ell} \prod_{\mu \in J}\left(1+a_{\mu}\right) \prod_{\nu \in J^{c}}\left(1-a_{\nu}\right) \\
= & (-1)^{\ell} i^{m-\ell} \sum_{J} \prod_{\mu \in J}\left(1+\frac{1-2 s_{\mu}}{1-2 s_{\mu}+2 s_{\mu}^{2}}+i \frac{-2 r_{\mu} s_{\mu}\left(-1+s_{\mu}\right)}{1-2 s_{\mu}+2 s_{\mu}^{2}}\right) \\
& \cdot \prod_{\nu \in J^{c}}\left(1-\frac{1-2 s_{v}}{1-2 s_{\nu}+2 s_{v}^{2}}-i \frac{-2 r_{\nu} s_{\nu}\left(-1+s_{\nu}\right)}{1-2 s_{v}+2 s_{v}^{2}}\right) \\
= & \frac{(-1)^{\ell} i^{m-\ell}}{\prod_{j=1}^{m} 1-2 s_{j}+2 s_{j}^{2}} \sum_{J} \prod_{\mu \in J} 2\left(1-2 s_{\mu}+s_{\mu}^{2}+i r_{\mu} s_{\mu}\left(1-s_{\mu}\right)\right) \\
& \cdot \prod_{\nu \in J^{c}} 2 s_{v}^{2}+2 i r_{\nu} s_{v}\left(-1+s_{\nu}\right) \\
= & \frac{2^{\ell}(-1)^{\ell} i^{m-\ell}}{S_{s}} \sum_{J} \prod_{\mu \in J}\left(1-s_{\mu}\right)\left(1-s_{\mu}+i r_{\mu} s_{\mu}\right) \prod_{\nu \in J^{c}} s_{v}\left(s_{\nu}+i r_{\nu}\left(s_{\nu}-1\right)\right)
\end{aligned}
$$

where

$$
S_{s}:=\prod_{j=1}^{m} 1-2 s_{j}+2 s_{j}^{2}
$$


Slightly rewriting it, we have

$$
\begin{aligned}
d_{\ell}= & \frac{(-2)^{\ell}}{S_{S}} \sum_{J}\left(\prod_{\nu \in J^{c}} s_{\nu}\right) i^{m-\ell}\left(\prod_{\nu \in J^{c}} s_{\nu}+i r_{\nu}\left(s_{\nu}-1\right)\right. \\
& \left.\cdot \prod_{\mu \in J}\left(1-s_{\mu}\right)\left(1-s_{\mu}+i r_{\mu} s_{\mu}\right)\right)
\end{aligned}
$$

and, in particular,

$$
d_{m}=\frac{(-2)^{m}}{S_{s}} \prod_{j=1}^{m}\left(1-s_{j}\right)\left(1-s_{j}+i r_{j} s_{j}\right)
$$

We also use

$$
S_{u}:=1-u^{2}+u^{4}, \quad S:=S_{s} S_{u}
$$

Observe that $S_{u} \geq 3 / 4$ (for $u \in[-1,1]$ ) and $1-2 s_{j}+2 s_{j}^{2} \geq 1 / 2, j=1, \ldots, m$ hence $S \geq 3 / 2^{m+2}$.

We rewrite the polynomials $U_{\ell}$ and $V_{\ell}$ 's using substitutions (38) and (39). So we introduce

$$
\begin{gathered}
R_{2 \ell+1}\left(u, s_{1}, s_{2}, \ldots, s_{m}, r_{1}, r_{2}, \ldots, r_{m}\right) \\
:=S U_{\ell}\left(\frac{u^{4}-3 u^{2}+1}{u^{4}-u^{2}+1},-\frac{2 u\left(u^{2}-1\right)}{u^{4}-u^{2}+1}, \frac{1-2 s_{1}}{1-2 s_{1}+2 s_{1}^{2}},-\frac{2 r_{1} s_{1}\left(-1+s_{1}\right)}{1-2 s_{1}+2 s_{1}^{2}},\right. \\
\left.\ldots, \frac{1-2 s_{m}}{1-2 s_{m}+2 s_{m}^{2}},-\frac{2 r_{m} s_{m}\left(-1+s_{m}\right)}{1-2 s_{m}+2 s_{m}^{2}}\right), \\
R_{2 \ell+2}\left(u, s_{1}, s_{2}, \ldots, s_{m}, r_{1}, r_{2}, \ldots, r_{m}\right) \\
:=S V_{\ell}\left(\frac{u^{4}-3 u^{2}+1}{u^{4}-u^{2}+1},-\frac{2 u\left(u^{2}-1\right)}{u^{4}-u^{2}+1}, \frac{1-2 s_{1}}{1-2 s_{1}+2 s_{1}^{2}},-\frac{2 r_{1} s_{1}\left(-1+s_{1}\right)}{1-2 s_{1}+2 s_{1}^{2}},\right. \\
\left.\ldots, \frac{1-2 s_{m}}{1-2 s_{m}+2 s_{m}^{2}},-\frac{2 r_{m} s_{m}\left(-1+s_{m}\right)}{1-2 s_{m}+2 s_{m}^{2}}\right)
\end{gathered}
$$

for $\ell=0,1, \ldots, m-1, m$. Note that $R_{j}$ 's are polynomials from $\mathbb{R}\left[u, s_{1}, r_{1}, \ldots, s_{m}, r_{m}\right]$. For simplicity, we define

$$
j_{0}:=\left\{\begin{array}{ll}
2 m+1, & \text { if } m \text { is even, } \\
2 m+2, & \text { if } m \text { is odd, }
\end{array} \quad j_{1}:= \begin{cases}2 m+2, & \text { if } m \text { is even } \\
2 m+1, & \text { if } m \text { is odd }\end{cases}\right.
$$

It is very important that $R_{j}, j=1,2, \ldots, 2 m$ do not have constant terms (because of the first factor on the right of (41))

$$
R_{j}(0, \ldots, 0)=0, \quad j=1,2, \ldots, 2 m
$$


In other words, using the substitution

$$
\mathbf{Z}: s_{1}=\ldots=s_{m}=0
$$

we can also write that

$$
\left.R_{j}\right|_{\mathbf{z}}=0, \quad j=1,2, \ldots, 2 m .
$$

The last two $R_{j}$ 's, namely $R_{2 m+1}$ and $R_{2 m+2}$ behave differently. The expressions (19) and (20) for $U_{m}$ and $V_{m}$ show that

- if $m$ is odd, then

$$
\begin{aligned}
U_{m} & =\frac{(-2)^{m+1}}{S_{s}}\left(\delta_{1}(1+\ldots)+\delta_{2} \cdot \ldots\right), \\
V_{m} & =\frac{2(-2)^{m}}{S_{s}}\left(\delta_{1} \cdot \ldots+\delta_{2}(1+\ldots)\right),
\end{aligned}
$$

- if $m$ is even, then

$$
\begin{aligned}
U_{m} & =\frac{2(-2)^{m}}{S_{s}}\left(\delta_{1} \cdot \ldots+\delta_{2}(1+\ldots)\right), \\
V_{m} & =\frac{2(-2)^{m}}{S_{s}}\left(\delta_{1}(1+\ldots)+\delta_{2} \cdot \ldots\right)
\end{aligned}
$$

where the ... stand for terms that are multiplied with an $s_{j}$.

Taking into account these four lines above and the substitutions (38) and (39), we can write

$$
\begin{aligned}
& R_{j_{0}} \mid \mathbf{z}=(-1)^{m+1} 2^{m+2} u\left(u^{2}-1\right), \\
& R_{j_{1}} \mid \mathbf{z}=2^{m+1}\left(u^{4}-3 u^{2}+1\right) .
\end{aligned}
$$

Hence $R_{j_{0}}$ has zero constant term and $R_{j_{1}}$ has non-zero constant term. These observations will be crucial for the argument later.

The system (31) with conditions (32) is equivalent to the system

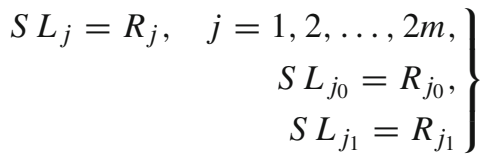

with the condition that $\left(u, s_{1}, \ldots, s_{m}, r_{1}, \ldots, r_{m}\right)$ are from the set

$$
\begin{array}{r}
W:=\left\{\left(u, s_{1}, \ldots, s_{m}, r_{1}, \ldots, r_{m}\right) \in \mathbb{R}^{2 m+1}:-1 \leq u \leq 1\right. \\
\left.0 \leq s_{1}, \ldots, s_{m} \leq 1,-1 \leq r_{1}, \ldots, r_{m} \leq 1\right\}
\end{array}
$$


We remark that $W$ is compact and $L_{j} \in \mathbb{R}\left[t_{1}, \ldots, t_{n}\right]$ and $R_{j} \in \mathbb{R}\left[u, s_{1}, r_{1}, \ldots, s_{m}, r_{m}\right]$.

For simplicity, we introduce $X_{1}=u, X_{1+j}=s_{j}, j=1,2, \ldots, m, X_{m+1+j}=r_{j}$, $j=1,2, \ldots, m$, and

$$
\mathbf{x}=\left(u, s_{1}, \ldots, s_{m}, r_{1}, \ldots, r_{m}\right)
$$

\subsection{Eliminating the Parametrizing Auxiliary Variables}

Since there is an identity submatrix within the matrix $A$ (coming from the coefficients of $L_{j}$ 's; see the definition of $A$, (33), and that of $L_{k}$ 's, (34) and (35)) there exists an invertible matrix $B_{2} \in \mathbb{R}^{2 n \times 2 n}$ such that with

$$
\left(\begin{array}{c}
\widetilde{L}_{1} \\
\widetilde{L}_{2} \\
\vdots \\
\widetilde{L}_{2 n}
\end{array}\right):=B_{2}\left(\begin{array}{c}
L_{1} \\
L_{2} \\
\vdots \\
L_{2 n}
\end{array}\right)
$$

we have

$$
\widetilde{L}_{j}= \begin{cases}0, & \text { if } j \in I_{2}, \\ t_{k}, & \text { if } j \in I_{1}, j=j(k) .\end{cases}
$$

This is a simple elimination on the left-hand sides (using row operations on $A$ instead of column operations which we used in Sect. 2.7). We transform the right-hand sides accordingly, hence we introduce $\widetilde{R}_{1}, \widetilde{R}_{2}, \ldots, \widetilde{R}_{2 n}$ as

$$
\left(\begin{array}{c}
\widetilde{R}_{1} \\
\widetilde{R}_{2} \\
\vdots \\
\widetilde{R}_{2 n}
\end{array}\right):=B_{2}\left(\begin{array}{c}
R_{1} \\
R_{2} \\
\vdots \\
R_{2 n}
\end{array}\right) .
$$

The system (47) with condition (48) is equivalent to

$$
\begin{aligned}
S t_{k} & =\widetilde{R}_{j(k)}(\mathbf{x}), \quad k=1,2, \ldots, n, \\
0 & =\widetilde{R}_{j}(\mathbf{x}), \quad j \in I_{2}
\end{aligned}
$$

with conditions (48), that is, when $\mathbf{x}=\left(u, s_{1}, \ldots, s_{m}, r_{1}, \ldots, r_{m}\right) \in W$.

Introduce

$$
\begin{aligned}
\widetilde{W}_{1} & :=\left\{(\mathbf{x}, t) \in \mathbb{R}^{3 n-1}: \mathbf{x} \in W, S_{t}=\widetilde{R}_{j(k)}(\mathbf{x}), k=1,2, \ldots, n\right\}, \\
\widetilde{W}_{2} & :=\left\{(\mathbf{x}, t) \in \mathbb{R}^{3 n-1}: \mathbf{x} \in W, 0=\widetilde{R}_{j}(\mathbf{x}), j \in I_{2}\right\}, \\
\widetilde{W} & :=\widetilde{W}_{1} \cap \widetilde{W}_{2}, \\
W_{2} & :=\left\{\mathbf{x} \in W:(\mathbf{x}, t) \in \widetilde{W}_{2} \text { for some } t \in \mathbb{R}^{n}\right\} .
\end{aligned}
$$


Using (52), we can express $t_{k}$ 's with $u, s_{1}, \ldots, s_{m}, r_{1}, \ldots, r_{m}$ as follows

$$
t_{k}=\frac{\widetilde{R}_{j(k)}(\mathbf{x})}{S}, \quad k=1, \ldots, n .
$$

Therefore if $\mathbf{x} \in W_{2}$, then there is a unique $t \in \mathbb{R}^{n}$ such that $(\mathbf{x}, t) \in \widetilde{W}$, because of the following. Note that $\widetilde{R}_{j}(\mathbf{x})$ are polynomials independent of $t_{1}, \ldots, t_{n}$. Furthermore, $1-u^{2}+u^{4} \geq 3 / 4$ when $u \in[-1,1]$ and $S \geq 3 / 2^{m+2}$ holds also. Therefore, for all possible $\mathbf{x} \in \bar{W}, \widetilde{R}_{j}(\underset{\widetilde{W}}{\mathbf{W}} / S(\mathbf{x})$ is continuous and bounded.

This implies that $\widetilde{W}$ is compact.

Hence, the $t$ 's coming from a solution are bounded, i.e. there is $M_{0}>0$ such that for all $t \in \mathbb{R}^{n}$ such that $(\mathbf{x}, t) \in \widetilde{W}$, we have $\left|t_{j}\right| \leq M_{0}, j=1,2, \ldots, n$.

\section{Application of the Positivstellensatz}

We are going to use a form of Positivstellensatz which can be found in the book of Prestel and Delzell [16]. Briefly, it is for sums of even powers and for compact (semialgebraic) sets.

First, we introduce our new set of notations. Then we apply the Positivstellensatz to find a solution. This indirect argument features a step-by-step simplification of the representation (61) provided by the Positivstellensatz. As the first step of simplification, we apply a substitution (63) which turns the representation into a univariate identity (68). Then a careful comparison of the leading terms and degrees leads to an even more simplified identity (69). Finally, exploiting the special structure of the equation (comparing (72) and (71)) leads to a contradiction. We remark that in this section we do not use the polynomials $c_{0}, c_{1}, \ldots, c_{m}$ from Sect. 2.3 (and we use $c_{0}, c_{1}$ as new symbols).

As the next step, we set

$$
N:=8
$$

Recall that $\mathbf{x}=\left(u, s_{1}, \ldots, s_{m}, r_{1}, \ldots, r_{m}\right)$ and for unifying the notation, we introduce the following:

$$
\begin{aligned}
h_{1}(\mathbf{x}) & :=\left(1+X_{1}^{2}\right)\left(1-X_{1}^{6}\right)=(-1) u^{8}-u^{6}+u^{2}+1, \\
h_{j+1}(\mathbf{x}) & :=X_{j+1}^{N-1}\left(1-X_{j+1}\right)=(-1) s_{j}^{N}+s_{j}^{N-1}, \quad j=1, \ldots, m, \\
h_{1+m+j}(\mathbf{x}) & :=1-X_{1+m+j}^{N}=(-1) r_{j}^{N}+1, \quad j=1, \ldots, m, \\
h_{2 m+2}(t) & :=M_{0}-\left(t_{1}^{N}+\ldots+t_{n}^{N}\right) .
\end{aligned}
$$

Put $N_{1}:=2 m+2$.

Note that $h_{1}(\mathbf{x}) \geq 0$ if and only if $u \in[-1,1]$ and $h_{1+j}(\mathbf{x}) \geq 0$ if and only if $s_{j} \in[0,1]$ and $h_{1+m+j}(\mathbf{x}) \geq 0$ if and only if $r_{j} \in[-1,1]$. Hence,

$$
\widehat{W}:=\left\{(\mathbf{x}, t) \in \mathbb{R}^{3 n-1}: h_{j}(\mathbf{x}, t) \geq 0, j=1, \ldots, N_{1}\right\}
$$


i.e. $\widehat{W}$ is a compact, semialgebraic set.

Introduce

$$
f(\mathbf{x}, t):=\sum_{j=1}^{m}\left(S L_{j}-R_{j}\right)^{2}+\left(S L_{j_{0}}-R_{j_{0}}\right)^{2}+\left(S L_{j_{1}}-R_{j_{1}}\right)^{2} .
$$

Obviously, $f(\mathbf{x}, t)=0$ at some $(\mathbf{x}, t) \in \widehat{W}$ if and only if $(\mathbf{x}, t)$ is a solution of (47) and also $f \geq 0$.

As the next step, we apply a form of Positivstellensatz, more precisely [16, Thm. 7.3.11, p. 174]. We verify the conditions now. The highest homogeneous parts of $h_{1}, \ldots, h_{N_{1}}$ are $-u^{8},-s_{1}^{8}, \ldots,-s_{m}^{8},-r_{1}^{8}, \ldots,-r_{m}^{8},-\left(t_{1}^{8}+\cdots+t_{n}^{8}\right)$ respectively, and it is easy to see that at least one of them is negative at every $(\mathbf{x}, t) \in \mathbb{R}^{3 n-1} \backslash\{(0, \ldots, 0)\}$, i.e. condition (7.3.11.1) is satisfied. Of course, they have the same degree, $N$, and (59) is compact. The theorem states that if $f>0$ on $\widehat{W}$, then $f$ is in the (Archimedean) module generated by $h_{1}, h_{2}, \ldots, h_{N_{1}}$ of level $N$, i.e.

$$
f(\mathbf{x}, t)=\sigma_{0}+\sigma_{1} h_{1}+\ldots+\sigma_{N_{1}} h_{N_{1}}
$$

where $\sigma_{0}, \sigma_{1}, \ldots, \sigma_{N_{1}}$ are sums of $N$-th powers, i.e. they are from

$$
\sum^{N}[\mathbf{x}, t]:=\left\{\sum_{j=1}^{k} P_{j}^{N}: P_{1}, \ldots, P_{k} \in \mathbb{R}[\mathbf{x}, t]\right\} .
$$

Indirectly, assume that $f>0$ on $\widehat{W}$ which implies that (61) holds.

As the next step, we apply a substitution for (61) to simplify it. The expression on the right of (61) after substitution $\mathbf{Y}$, where

$$
\mathbf{Y}: s_{1}=\ldots=s_{m}=0, r_{1}=\ldots=r_{m}=0, t_{1}=\ldots=t_{n}=0
$$

has the following structure. Obviously,

$$
\begin{aligned}
h_{1}(u):=h_{1} \mid \mathbf{Y} & =h_{1}(u)=\left(u^{2}+1\right)\left(1-u^{6}\right), \\
h_{1+j} \mid \mathbf{Y} & =0, \quad j=1,2, \ldots, m, \\
h_{1+m+j} \mid \mathbf{Y} & =1, \quad j=1,2, \ldots, m, \\
h_{2+2 m} \mid \mathbf{Y} & =M_{0} \text { (const). }
\end{aligned}
$$

Also, if $\sigma \in \sum^{N}[\mathbf{x}, t]$, then $\left.\sigma\right|_{\mathbf{Y}} \in \sum^{N}[u]$. Therefore the right-hand side will have this form:

$$
\sigma_{0}+\left.\sum_{j=1}^{N_{1}} \sigma_{j} h_{j}\right|_{\mathbf{Y}}=\sigma_{0}+\sigma_{1}\left(u^{2}+1\right)\left(1-u^{6}\right)
$$

where slightly abusing the notation, we write $\left.\sigma\right|_{\mathbf{Y}}=\sigma$. 
The substitution simplifies greatly the left-hand side of (61)

$$
\begin{aligned}
f_{1}(u):=\left.f\right|_{\mathbf{Y}} & =\left(S L_{j_{0}}-R_{j_{0}} \mid \mathbf{Y}\right)^{2}+\left(S L_{j_{1}}-R_{j_{1}} \mid \mathbf{Y}\right)^{2} \\
& =\left(2^{m+2} u\left(u^{2}-1\right)\right)^{2}+\left(2^{m+1}\left(u^{4}-3 u^{2}+1\right)\right)^{2}
\end{aligned}
$$

where we used (45), (46) and also (44).

As the next step, we rewrite $u^{2}+1$ with a result of Berr and Wörmann. Obviously, $u^{2}+1$ is strictly positive on $[-1,1]$, so it can be written as

$$
u^{2}+1=\tau_{0}+\tau_{1}\left(1-u^{6}\right)+\cdots+\tau_{7}\left(1-u^{6}\right)^{7}
$$

where $\tau_{0}, \tau_{1}, \ldots, \tau_{7} \in \sum^{N}[u]$ (recall $N=8$ ). This representation follows from [2, Ex. 4.5, p. 834]. It would be interesting to establish this expansion directly.

Using (66), we rewrite the right-hand side of (64):

$$
\begin{aligned}
\sigma_{0}+ & \sigma_{1}\left(u^{2}+1\right)\left(1-u^{6}\right) \\
& =\sigma_{0}+\left(\sigma_{1} \tau_{0}\right)\left(1-u^{6}\right)+\cdots+\left(\sigma_{1} \tau_{6}\right)\left(1-u^{6}\right)^{7}+\left(\sigma_{1} \tau_{7}\right)\left(1-u^{6}\right)^{8} \\
& =\left(\sigma_{0}+\left(\sigma_{1} \tau_{7}\right)\left(1-u^{6}\right)^{8}\right)+\left(\sigma_{1} \tau_{0}\right)\left(1-u^{6}\right)+\cdots+\left(\sigma_{1} \tau_{6}\right)\left(1-u^{6}\right)^{7} \\
& =\widetilde{\sigma}_{0}+\widetilde{\sigma}_{1}\left(1-u^{6}\right)+\cdots+\widetilde{\sigma}_{7}\left(1-u^{6}\right)^{7}
\end{aligned}
$$

where

$$
\begin{aligned}
\widetilde{\sigma}_{0} & :=\sigma_{0}+\left(\sigma_{1} \tau_{7}\right)\left(1-u^{6}\right)^{8} \in \sum^{N}[u], \\
\widetilde{\sigma}_{1} & :=\sigma_{1} \tau_{0} \in \sum^{N}[u], \\
& \vdots \\
\widetilde{\sigma}_{7} & :=\sigma_{1} \tau_{6} \in \sum^{N}[u] .
\end{aligned}
$$

As the next step, we collect the results of substitution and simplification. Using (61), (66) and (67), the right-hand side is relatively simple, while regarding the left-hand side, we use (65) and we write

$$
f_{1}(u)=\widetilde{\sigma}_{0}+\widetilde{\sigma}_{1}\left(1-u^{6}\right)+\cdots+\widetilde{\sigma}_{7}\left(1-u^{6}\right)^{7} .
$$


To compare the two sides, we need the powers of $1-u^{6}$ :

$$
\begin{aligned}
\left(1-u^{6}\right)^{1}= & (-1) u^{6}+1, \\
\left(1-u^{6}\right)^{2}= & u^{12}-2 u^{6}+1=u^{4} u^{N}-2 u^{6}+1, \\
\left(1-u^{6}\right)^{3}= & (-1) u^{18}+3 u^{12}-3 u^{6}+1 \\
= & (-1) u^{2}\left(u^{2}\right)^{N}+3 u^{4} u^{N}-3 u^{6}+1, \\
\left(1-u^{6}\right)^{4}= & u^{24}-4 u^{18}+6 u^{12}-4 u^{6}+1 \\
= & \left(u^{3}\right)^{N}-4 u^{2}\left(u^{2}\right)^{N}+6 u^{4} u^{N}-4 u^{6}+1, \\
\left(1-u^{6}\right)^{5}= & (-1) u^{30}+5 u^{24}-10 u^{18}+10 u^{12}-5 u^{6}+1 \\
= & (-1) u^{6}\left(u^{3}\right)^{N}+5\left(u^{3}\right)^{N}-10 u^{2}\left(u^{2}\right)^{N}+10 u^{4} u^{N}-5 u^{6}+1, \\
\left(1-u^{6}\right)^{6}= & u^{36}-6 u^{30}+15 u^{24}-20 u^{18}+15 u^{12}-6 u^{6}+1 \\
= & u^{4}\left(u^{4}\right)^{N}-6 u^{6}\left(u^{3}\right)^{N}+15\left(u^{3}\right)^{N} \\
& -20 u^{2}\left(u^{2}\right)^{N}+15 u^{4} u^{N}-6 u^{6}+1, \\
\left(1-u^{6}\right)^{7}= & (-1) u^{42}+7 u^{36}-21 u^{30}+35 u^{24}-35 u^{18}+21 u^{12}-7 u^{6}+1 \\
= & (-1) u^{2}\left(u^{5}\right)^{N}+7 u^{4}\left(u^{4}\right)^{N}-21 u^{6}\left(u^{4}\right)^{N} \\
& +35\left(u^{3}\right)^{N}-35 u^{2}\left(u^{2}\right)^{N}+21 u^{4} u^{N}-7 u^{6}+1 .
\end{aligned}
$$

We investigate the degrees and the leading terms in (68). On the left-hand side, $\operatorname{deg} f_{1}=N=8$. The right-hand side is more involved. We remark that $\operatorname{deg} \widetilde{\sigma}_{j}=N k_{j}$ for some $k_{j} \in \mathbb{N}$ and the leading coefficient $\operatorname{lc}\left(\widetilde{\sigma}_{j}\right)$ of $\widetilde{\sigma}_{j}$ is positive. We investigate the degrees of $\widetilde{\sigma}_{j}\left(1-u^{6}\right)^{j}, j=0,1, \ldots, 7$ modulo $N$. Consider the following groups of powers: $\{0,4\}$ and $\{1,5\}$ and $\{2,6\}$ and $\{3,7\}$. In each group they have the same degrees $\bmod N$ and the same signs of leading coefficients, e.g. if we take $\{1,5\}$, then $\operatorname{deg} \widetilde{\sigma}_{1}\left(1-u^{6}\right)^{1}=N k_{1}+6, \operatorname{deg} \widetilde{\sigma}_{5}\left(1-u^{6}\right)^{5}=N k_{5}+30=N\left(k_{5}+3\right)+6$ and the signs of the leading coefficients are the same sign $\operatorname{lc}\left(\widetilde{\sigma}_{1}\left(1-u^{6}\right)^{1}\right)=\operatorname{sign} \operatorname{lc}\left(\widetilde{\sigma}_{5}\left(1-u^{6}\right)^{5}\right)=$ -1 . Since the signs are the same, the leading terms in the same group cannot cancel. Also, since the degrees of $\widetilde{\sigma}_{j}\left(1-u^{6}\right)^{j}$ are different modulo $N=8$, the leading terms of different groups cannot cancel either. Hence

$$
\operatorname{deg}\left(f_{1}\right)=\max \left(\operatorname{deg} \widetilde{\sigma}_{0}, \operatorname{deg} \widetilde{\sigma}_{1}\left(1-u^{6}\right), \operatorname{deg} \widetilde{\sigma}_{2}\left(1-u^{6}\right)^{2}, \ldots, \operatorname{deg} \widetilde{\sigma}_{7}\left(1-u^{6}\right)^{7}\right) .
$$

Taking into account that $\operatorname{deg}\left(f_{1}\right)=8$, this can happen only when

$$
\widetilde{\sigma}_{2}=\ldots=\tilde{\sigma}_{7}=0 \quad \text { and } \quad \operatorname{deg} \widetilde{\sigma}_{1}=0
$$

i.e. $\widetilde{\sigma}_{1}$ is a constant.

Finally, we obtain that

$$
f_{1}=\widetilde{\sigma}_{0}+c_{1}\left(1-u^{6}\right)
$$


where $c_{1} \geq 0$.

So we have

$$
f_{1}(u)=\sum_{j=1}^{N_{2}} A_{j}^{N}(u)+c_{1}\left(1-u^{6}\right)
$$

where $N_{2}$ is a positive integer and $A_{j}(u)$ are polynomials. Again, degree considerations show that the $A_{j}$ 's must be constant or linear polynomials, so we can write

$$
f_{1}(u)=c_{0}+\sum_{j=1}^{N_{3}} \lambda_{j}\left(u-\zeta_{j}\right)^{N}+c_{1}\left(1-u^{6}\right)
$$

where $\lambda_{j}>0, \zeta_{j} \in \mathbb{R}$ are pairwise different and $c_{0} \in \mathbb{R}$ and $N_{3} \leq N_{2}$.

We finally reach a contradiction by showing that (71) cannot hold. Note that

$$
\begin{aligned}
f_{1}(u) & =4^{m+1}\left(4 u^{2}(u-1)^{2}(u+1)^{2}+\left(u^{4}-3 u^{2}+1\right)^{2}\right) \\
& =4^{m+1}\left(u^{8}-2 u^{6}+3 u^{4}-2 u^{2}+1\right)
\end{aligned}
$$

hence $f_{1}(\cdot)$ is even, $f_{1}(-u)=f_{1}(u)$. Exploiting this (and that $N$ is even), we write

$$
f_{1}(u)=\frac{f_{1}(u)+f_{1}(-u)}{2}=c_{0}+\sum_{j=1}^{N_{3}} \lambda_{j} \frac{\left(u-\zeta_{j}\right)^{N}+\left(u+\zeta_{j}\right)^{N}}{2}+c_{1}\left(1-u^{6}\right) .
$$

Observe that all coefficients of $\left(\left(u-\zeta_{j}\right)^{N}+\left(u+\zeta_{j}\right)^{N}\right) / 2$ are non-negative (e.g. the coefficient of $u^{2}$ is $28 \zeta_{j}^{6}$ ). Therefore the coefficient of $u^{2}$ is $4^{m+1}(-2)<0$ (according to the left-hand side of (71)) and it is $\sum_{j=1}^{N_{3}} \lambda_{j} \cdot 28 \zeta_{j}^{6} \geq 0$ (according to the right-hand side of (71)). This gives a contradiction.

Therefore, we have $f \ngtr 0$. Obviously $f \geq 0$. These two imply that $f$ must have a zero on $\widehat{W},(\mathbf{x}, t) \in \widehat{W}$ with $f(\mathbf{x}, t)=0$, so the system (52) and (53) with $\mathbf{x} \in W$ has a solution. In turn, this implies that $\widetilde{W} \neq 0$ which leads to the fact that (47) has a solution from $W$ (see (48)), which implies that (31) has a solution under the conditions (32), that is, (29) holds which is equivalent to the assertion of Theorem 1. Therefore the proof is complete. 


\section{Some Technical Lemmas}

Lemma 1 Let $W \in \mathbb{C}, W \neq 0, \gamma \in \mathbb{C},|\gamma|=1$. Then there exists $f \in \mathbb{C},|f|=1$ such that

$$
i \frac{\bar{W}+\gamma W}{\bar{W}-\gamma W}=\frac{f(\bar{W}+\gamma W)}{\frac{f}{i}(\bar{W}-\gamma W)}
$$

and the numerator and denominator on the right are real, i.e.

$$
\operatorname{Im}(f(\bar{W}+\gamma W))=0, \quad \operatorname{Im}\left(\frac{f}{i}(\bar{W}-\gamma W)\right)=0 .
$$

In particular, $f=\sqrt{\gamma}$ will do. Furthermore, a similar identity also holds: there exists $f \in \mathbb{C},|f|=1$ such that

$$
i \frac{\bar{W}-\gamma W}{\bar{W}+\gamma W}=\frac{f(\bar{W}-\gamma W)}{\frac{f}{i}(\bar{W}+\gamma W)}
$$

and again, the numerator and the denominator on the right are real. In this case, $f=i / \sqrt{\gamma}$ is a good choice.

Proof We use a separate set of notation in this proof.

To see the first assertion, write $W, \gamma$ and $f$ in polar form in this proof: $W=r e^{i \omega}$, where $r>0, \gamma=e^{i \alpha}$ and $f=e^{i \varphi}$. We also have

$$
\begin{aligned}
\operatorname{Im}(f(\bar{W}+\gamma W)) & =r \operatorname{Im}\left(e^{i(\varphi-\omega)}+e^{i(\varphi+\omega+\alpha)}\right) \\
& =r(\sin (\varphi-\omega)+\sin (\varphi+\omega+\alpha)) \\
& =2 r \sin \left(\varphi+\frac{\alpha}{2}\right) \cos \left(\omega+\frac{\alpha}{2}\right),
\end{aligned}
$$

and similarly for the denominator,

$$
\begin{aligned}
\operatorname{Im}\left(\frac{f}{i}(\bar{W}-\gamma W)\right) & =r \operatorname{Im}\left(e^{i(-\pi / 2+\varphi-\omega)}-e^{i(-\pi / 2+\varphi+\omega+\alpha)}\right) \\
& =r\left(\sin \left(-\frac{\pi}{2}+\varphi-\omega\right)-\sin \left(-\frac{\pi}{2}+\varphi+\omega+\alpha\right)\right) \\
& =-2 r \sin \left(\omega+\frac{\alpha}{2}\right) \cos \left(-\frac{\pi}{2}+\varphi+\frac{\alpha}{2}\right) \\
& =-2 r \sin \left(\omega+\frac{\alpha}{2}\right) \sin \left(\varphi+\frac{\alpha}{2}\right) .
\end{aligned}
$$

So we have to find $\varphi$ for the given $\alpha$ and $\omega$ such that

$$
\sin \left(\varphi+\frac{\alpha}{2}\right) \cos \left(\omega+\frac{\alpha}{2}\right)=0 \text { and } \sin \left(\omega+\frac{\alpha}{2}\right) \sin \left(\varphi+\frac{\alpha}{2}\right)=0 .
$$


So $\varphi=-\alpha / 2$ will do.

For the second assertion, a similar argument yields that $\varphi=(\pi-\alpha) / 2$ will do.

Lemma 2 Let $P, Q$ be complex polynomials without common zeros. Assume that $H(u)=P(u) / Q(u)$ is a real rational function, i.e. if $u \in \mathbb{R}$ and $H(u)$ is finite, then $H(u) \in \mathbb{R}$. Also assume that the leading coefficients of $P$ and $Q$ are real. Then all the coefficients of $P$ and $Q$ are real.

Proof We prove it by induction as follows. Write $P(u)=a u^{n}+P_{1}(u)$ and $Q(u)=$ $b u^{m}+Q_{1}(u)$ where $\operatorname{deg}\left(P_{1}\right)<\operatorname{deg}(P)$ and $\operatorname{deg}\left(Q_{1}\right)<\operatorname{deg}(Q)$. By the assumptions, $a, b \in \mathbb{R}, a \neq 0, b \neq 0$.

If $n \geq m>0$, we can write

$$
H(u)=\frac{P(u)}{Q(u)}=\frac{a u^{n}+P_{1}(u)}{b u^{m}+Q_{1}(u)}=\frac{a}{b} u^{n-m}+\frac{P(u)-\frac{a}{b} u^{n-m} Q(u)}{Q(u)}
$$

which implies that with $P_{2}(u):=P(u)-\frac{a}{b} u^{n-m} Q(u), P_{2}(u) / Q(u)$ is a real rational function. Note that $n_{1}:=\operatorname{deg}\left(P_{2}\right)<\operatorname{deg}(P)=n$. Denote the leading coefficient of $P_{2}$ by $c, c \in \mathbb{C}, c \neq 0$. It is standard to see that

$$
\lim _{u \rightarrow \infty} \frac{u^{m}}{u^{n_{1}}} \frac{P_{2}(u)}{Q(u)}=\frac{c}{b}
$$

where the left-hand side is real and $b$ on the right is also real. Hence the leading coefficient $c$ of $P_{2}$ is real.

If $m>n>0$, then consider $1 / H(u)=Q(u) / P(u)$ which is again a real rational function.

If $m=0$, then $H(u)=P(u) / Q(u)$ is actually a polynomial. Also, $Q(u)=Q(0) \in$ $\mathbb{R}$, and hence $H(u)$ is a real polynomial, that is, if $u \in \mathbb{R}$, then $H(u) \in \mathbb{R}$. It is then standard that the polynomial $H(u)$ must have real coefficients.

Finally, if $n=0$, then consider $1 / H(u)$ and this way we reduce this case to the case discussed in the previous paragraph.

We also need the following lemma ( [17, Lem. 2])

Lemma 3 For any sequence $\left(B_{n}\right)$ of Blaschke products of degree $m$ there exist a Blaschke product $B$ of degree $k \leq m$ and a subsequence of $\left(B_{n}\right)$ which converges to $B$ locally uniformly on a set which contains all points of $\mathbb{D} \cup \mathbb{T}$ with the possible exception of at most $m-k$ boundary points.

Acknowledgements The research of Sergei Kalmykov was supported by Moscow Center for Fundamental and Applied Mathematics, Agreement with the Ministry of Science and Higher Education of the Russian Federation, No. 075-15-2019-1623 and the Russian Science Foundation grant 19-71-30004 (Section 2). Béla Nagy was supported by DAAD-TKA Research Project "Harmonic Analysis and Extremal Problems" \#308015. The authors thank the referee for careful reading and helpful suggestions.

Funding Open access funding provided by University of Szeged. 
Open Access This article is licensed under a Creative Commons Attribution 4.0 International License, which permits use, sharing, adaptation, distribution and reproduction in any medium or format, as long as you give appropriate credit to the original author(s) and the source, provide a link to the Creative Commons licence, and indicate if changes were made. The images or other third party material in this article are included in the article's Creative Commons licence, unless indicated otherwise in a credit line to the material. If material is not included in the article's Creative Commons licence and your intended use is not permitted by statutory regulation or exceeds the permitted use, you will need to obtain permission directly from the copyright holder. To view a copy of this licence, visit http://creativecommons.org/licenses/by/4.0/.

\section{References}

1. Agler, J., Lykova, Z.A., Young, N.J.: Finite Blaschke products and the construction of rational $\Gamma$-inner functions. J. Math. Anal. Appl. 447(2), 1163-1196 (2017). https://doi.org/10.1016/j.jmaa.2016.10. 035

2. Berr, R., Wörmann, T.: Positive polynomials and tame preorderings. Math. Z. 236(4), 813-840 (2001). https://doi.org/10.1007/PL00004853

3. Cantor, D.G., Phelps, R.R.: An elementary interpolation theorem. Proc. Am. Math. Soc. 16, 523-525 (1965). https://doi.org/10.2307/2034689

4. Daepp, U., Gorkin, P., Shaffer, A., Voss, K.: Finding ellipses. In: Carus Mathematical Monographs, vol. 34. MAA Press, Providence (2018). What Blaschke products, Poncelet's theorem, and the numerical range know about each other. https://doi.org/10.1090/car/034

5. Garcia, S.R., Mashreghi, J., Ross, W.T.: Finite Blaschke products and their connections. Springer, Cham (2018). https://doi.org/10.1007/978-3-319-78247-8

6. Glader, C.: Rational unimodular interpolation on the unit circle. Comput. Methods Funct. Theory 6(2), 481-492 (2006). https://doi.org/10.1007/BF03321625

7. Glader, C.: Minimal degree rational unimodular interpolation on the unit circle. Electron. Trans. Numer. Anal. 30, 88-106 (2008)

8. Glader, C., Lindström, M.: Finite Blaschke product interpolation on the closed unit disc. J. Math. Anal. Appl. 273(2), 417-427 (2002). https://doi.org/10.1016/S0022-247X(02)00249-4

9. Gorkin, P., Mortini, R.: Radial limits of interpolating Blaschke products. Math. Ann. 331(2), 417-444 (2005). https://doi.org/10.1007/s00208-004-0588-0

10. Gorkin, P., Rhoades, R.C.: Boundary interpolation by finite Blaschke products. Constr. Approx. 27(1), 75-98 (2008). https://doi.org/10.1007/s00365-006-0646-3

11. Hjelle, G.A.: Approximations by interpolating Blaschke products. Ph.D. thesis, Norwegian University of Science and Technology, Trondheim (2006). http://citeseerx.ist.psu.edu/viewdoc/download? doi=10.1.1.527.9301\&rep=rep1\&type $=$ pdf

12. Hjelle, G.A.: Unimodular functions and interpolating Blaschke products. Proc. Am. Math. Soc. 134(1), 207-214 (2006). https://doi.org/10.1090/S0002-9939-05-07968-2

13. Hjelle, G.A.: Constructing interpolating Blaschke products with given preimages. Comput. Methods Funct. Theory 7(1), 43-54 (2007). https://doi.org/10.1007/BF03321630

14. Horn, R.A., Johnson, C.R.: Matrix Analysis, 2nd edn. Cambridge University Press, Cambridge (2013)

15. Jones, W.B., Ruscheweyh, S.: Blaschke product interpolation and its application to the design of digital filters. Constr. Approx. 3(4), 405-409 (1987). https://doi.org/10.1007/BF01890578

16. Prestel, A., Delzell, C.N.: Positive polynomials. In: Springer Monographs in Mathematics. Springer, Berlin (2001). From Hilbert's 17th problem to real algebra.https://doi.org/10.1007/978-3-662-046487

17. Semmler, G., Wegert, E.: Boundary interpolation with Blaschke products of minimal degree. Comput. Methods Funct. Theory 6(2), 493-511 (2006). https://doi.org/10.1007/BF03321626

18. Semmler, G., Wegert, E.: Finite Blaschke products with prescribed critical points, Stieltjes polynomials, and moment problems. Anal. Math. Phys. 9(1), 221-249 (2019). https://doi.org/10.1007/s13324-0170193-5

19. Younis, R.: Interpolation by a finite Blaschke product. Proc. Am. Math. Soc. 78(3), 451-452 (1980).https://doi.org/10.2307/2042343

Publisher's Note Springer Nature remains neutral with regard to jurisdictional claims in published maps and institutional affiliations. 